\title{
TRANSFORMATION GROUPS ON A HOMOLOGICAL MANIFOLD
}

BY

C. T. YANG

1. Introduction. In a recent paper of Montgomery-Samelson-Zippin [1], the following theorem is proved. If a compact Lie group acts as a topological transformation group on an $n$-dimensional manifold such that the highest dimension of any orbit is $r$, then the union of all the orbits of dimension $\leqq k, 0 \leqq k<r$, is a closed set of dimension $\leqq n-r+k-1$. Hence the singular set, i.e., the union of all the orbits not of the highest dimension, is a closed set of dimension $\leqq n-2$. The purpose of the present paper is to generalize this theorem to a homological manifold. This generalization together with some other results of this paper will be used in a further study [2] of transformation groups on a homological manifold.

Let $X$ be an $n$-dimensional manifold and let $G$ be a compact Lie group acting topologically on $X$. For each $x \in X, G_{x}$ denotes the isotropic subgroup of $G$ at $x$. It is known [3] that every point $p$ of $X$ has an open neighborhood $U$ such that there is a subset $Y$ of $U$ containing $p$ and an open cell $Q$ in $G$ containing the identity such that (i) whenever $g \in G$ and $x \in Y, g(x) \in Y$ if and only if $g \in G_{p}$ and (ii) $(g, x) \rightarrow g(x)$ defines a homeomorphism of $Q \times Y$ on to $U . Y$ is called a slice at $p$ and may be assumed to be connected. As one can easily see, $G_{p}$ may be regarded as a transformation group on $Y$ and its orbit space coincides with that of $G(Y)$ acted on by $G$. Moreover, an orbit in $G(Y)$ is of the same type as $G(p)$ if and only if it intersects $Y$ at a single point. Therefore certain properties of the set of the orbits in $G(Y)$ which are of the same type as $G(p)$, can be obtained by studying the fixed point set of $G_{p}$ in $Y$.

If $G$ acts differentiably on $X$, we may have open cells as slices [4] and then the proof of the cited theorem can be greatly simplified by using the remark of the preceding paragraph. For the nondifferentiable case it is not known whether $Y$ is a manifold, as it is still an open question whether a topological space is a $k$-dimensional manifold if the product of the space and the real line is known to be an $(n+1)$-dimensional manifold. However, $Y$ can be shown to be a homological manifold. Therefore it seems more natural to begin with a homological manifold $X$ and then to proceed to the proof just as in the differential case. Moreover, it is pointed out in [1] that the original proof depends only on the local homology property of a manifold, and this gives us another justification to deal with homological manifolds rather than ordinary manifolds.

Received by the editors July 2, 1956. 
By a homological $n$-manifold we mean a connected, finite-dimensional, locally compact Hausdorff space which is of type $P_{n}$ in the sense of Smith [5], where the dimension is defined to be the highest Lebesgue covering dimension of any compact subset of the space. But we can not use the group $\mathfrak{C}_{q}$ of integers modulo a fixed prime number $q$ as the coefficient group as Smith did in [5], because we need the property $P_{n}$ with respect to various $\mathfrak{S}_{q}$ at the same time. It will be seen later that we can meet this requirement by using the group $\mathfrak{B}$ of reals modulo 1 as coefficient group. Another reason for using $\mathfrak{P}$ as coefficient group is that it follows from [6] that the dimension of such a homological $n$-manifold is exactly equal to $n$. Note that the homological manifolds in our sense and the generalized manifolds defined in Wilder [7] are alike to a great extent.

To conclude the introduction the author wishes to express his gratitude to Professor Montgomery for his valuable suggestions and encouragement when this paper was prepared.

2. A homology theory on $\mathfrak{A}_{L C}$. We shall use a compact abelian group $\mathfrak{C}$ as coefficient group which of course can be the additive group $\mathfrak{B}$ of reals modulo 1 or the additive group $\mathfrak{E}_{q}$ of integers modulo a prime number $q$. Let $\mathfrak{A}_{L C}$ be the admissible category defined to consist of all the pairs $(X, A)$ such that $X$ is a locally compact Hausdorff space and $A$ is a closed subset of $X$, and to consist of all the maps $f$ of such pairs such that the inverse image of any compact set under $f$ is compact. Let $\mathfrak{A}_{C}$ be the subcategory of $\mathfrak{A}_{L C}$ which consists of all the compact pairs and all the maps of such pairs. By the onepoint-compactification process, we can use the Cech homology theory on $\mathfrak{A}_{C}$ to define a homology theory on $\mathfrak{A}_{L C}$ [8]. This homology theory on $\mathfrak{A}_{L C}$ satisfies the Eilenberg-Steenrod axioms and the continuity axiom [8] and will be used throughout this paper.

Whenever $(X, A) \in \mathfrak{A}_{L C}, H_{k}(X, A$; (S) denotes the $k$ th homology group of $(X, A)$, i.e., the $k$ th Cech homology group of $(X \cup \omega, A \cup \omega)$ with coefficients in $\mathcal{E}$, where $X \cup \omega$ is the one-point-compactification of $X . \partial$ denotes the boundary operator of a homology sequence and $\Delta$ denotes the boundary operator of a Mayer-Vietoris sequence.

If $f:(X, A) \rightarrow(Y, B)$ is a map in $\mathfrak{A}_{L C}$, the notation

$$
H_{k}\left(X, A ;(\mathfrak{S}) \stackrel{f_{*}}{\rightarrow} H_{k}(Y, B ; \mathfrak{S})\right.
$$

means the homomorphism of $H_{k}\left(X, A\right.$; (5) into $H_{k}(Y, B$; $(5)$ induced by $f$. If in particular $f$ is the inclusion map, we write only

$$
H_{k}\left(X, A ;(\mathfrak{S}) \rightarrow H_{k}(Y, B ; \text { ( })\right.
$$

and call it the natural homomorphism of $H_{k}(X, A$; $\mathfrak{S})$ into $H_{k}(Y, B$; $\mathfrak{S})$. Whenever $S$ is a subset or an element of $H_{k}(X, A ; \mathfrak{C}), S \mid(Y, B)$ will denote the natural homomorphic image of $S$ in $H_{k}(Y, B$; $\mathfrak{(})$. Any arrow to which a symbol $\approx$ is attached will indicate an isomorphism onto. 
Lemma 1. Let $(X, A) \in \mathfrak{A}_{L C}$ and let $e \in H_{k}(X, A$; $\mathfrak{E})$. Let $Y$ be a closed subset of $X$. Then $e \mid(X, Y \cup A)=0$ if and only if $e \in H_{k}(Y \cup A, A$; (E) $) \mid(X, A)$. Moreover, there exists a minimal $Y$ satisfying these conditions, and such a set $Y$ is contained in $(X-A)^{-}$.

Lemma 2. Let $(X, A) \in \mathfrak{A}_{L C}$ and let $Y$ be a closed $t$-cell with boundary $B$. Then there is a natural isomorphism $i$ of $H_{k}\left(X, A\right.$; (5) onto $H_{k+t}(X \times Y,(X \times B)$ $\cup(A \times Y)$; (5). If $f:(X, A) \rightarrow\left(X^{\prime}, A^{\prime}\right)$ is a map in $\mathfrak{A}_{L C}$ and $j:(Y, B) \rightarrow(Y, B)$ is the identity map, then the commutativity relation $(f \times j)_{*} i=i f_{*}$ holds in the diagram

$$
\begin{aligned}
& H_{k}(X, A ; \mathfrak{s}) \stackrel{i}{\rightarrow} H_{k+t}(X \times Y,(X \times B) \cup(A \times Y) ;(\mathfrak{S})
\end{aligned}
$$

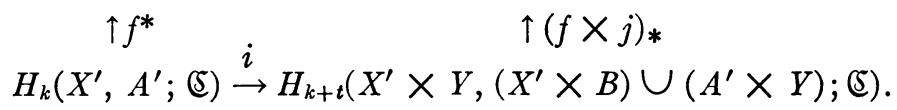

3. The property $P_{n}$. A subset of a locally compact Hausdorff space is called bounded if its closure is compact. If $X$ is a locally compact Hausdorff space and $x$ is a point of $X$, a neighborhood of $x$ (in $X$ ) will always mean a bounded open subset of $X$ containing $x$.

Definition. Let $X$ be a locally compact Hausdorff space and let $n$ be a non-negative integer. A non-null bounded open subset $U$ of $X$ and a subgroup $S$ of $H_{n}(X, X-U$; () are said to form a fundamental (ㄷ, $n)$-pair $(U, S)$ if the following conditions are satisfied.

(i) $S$ is isomorphic to (5.

(ii) Whenever $W$ is a non-null open subset of $U$, the natural homomorphism of $H_{n}(X, X-U$; $\mathfrak{E})$ into $H_{n}(X, X-W$; $\mathfrak{E})$ maps $S$ isomorphically onto $S \mid(X, X-W)$.

(iii) Whenever $y$ is a point of $U$ and $V$ is a neighborhood of $y$, there is a neighborhood $W$ of $y$ contained in $U \cap V$ and such that

$$
H_{n}(X, X-V ;(5)|(X, X-W) \subset S|(X, X-W)
$$

and for $k \neq n$,

$$
H_{k}(X, X-V ; \mathfrak{S}) \mid(X, X-W)=0 .
$$

REMARK 1. If $(U, S)$ is a fundamental $(\mathfrak{S}, n)$-pair and $Q$ is a non-null open subset of $U$, then $(Q, S \mid(X, X-Q))$ is also a fundamental (א, $n)$-pair.

Definition. Let $X$ be a locally compact Hausdorff space. At a point $x$ of $X, X$ possesses the property $P_{n}(\mathfrak{S})$ if there is a fundamental $(\mathfrak{S}, n)$-pair $(U, S)$ with $U$ containing $x . X$ is of type $P(\mathbb{E})$ if $X$ possesses the property $P_{n}(\mathfrak{E})$ at every point $x, n$ depending on $x$. If in particular $n$ is a constant over $X, X$ is of type $P_{n}(\mathfrak{S})$.

For $\mathfrak{S}=\mathfrak{S}_{q}$ these two definitions are due to Smith [5].

REMARK 2. If $X$ is connected and of type $P(\mathfrak{E})$, then $X$ is of type $P_{n}$ (ङ) for some integer $n$. 
As a consequence of these definitions and Lemma 2, we have

Lemma 3. Let $X$ be a locally compact Hausdorff space and let $R^{t}$ be the euclidean $t$-space. Let $U$ be a non-null bounded open subset of $X$ and let $V$ be a bounded open $t$-cell in $R^{t}$. Let $S$ be a subgroup of $H_{n}(X, X-U$; $\mathfrak{E})$ and let $T$ be a natural isomorphic image of $S$ in $H_{n+t}\left(X \times R^{t},\left(X \times R^{t}\right)-(U \times V)\right.$; (5); then $(U, S)$ is a fundamental $(\mathbb{S}, n)$-pair if and only if $(U \times V, T)$ is a fundamental $(\mathcal{E}, n+t)$-pair. Hence $X$ is of type $P_{n}(\mathbb{E})$ if and only if $X \times R^{t}$ is of type $P_{n+t}(\mathbb{S})$.

Definition. A locally compact Hausdorff space $X$ is of dimension $\leqq k$ if the Lebesgue covering dimension of every compact subset of $X$ is $\leqq k$.

A characterization of the dimension is [6]

Lemma 4. A finite-dimensional locally compact Hausdorff space $X$ is of dimension $k$ if and only if $k$ is the largest integer such that for some compact subsets $M, N$ of $X, M \supset N$ and $H_{k}(M, N ; \mathfrak{B}) \neq 0$.

Remark 3. Because of Lemma 1 , the compact subsets $M, N$ in Lemma 4 can be so chosen that for some nonzero element $e$ of $H_{k}(M, N ; \mathfrak{B}), e \mid(M, K \cup N)$ $\neq 0$ whenever $K$ is a proper compact subset of $(M-N)^{-}$.

Corollary. In a finite-dimensional locally compact Hausdorff space $X$ there is a point $x$ such that every neighborhood of $x$ is of the same dimension as $X$.

Because of Lemma 4, we have

Lemma 5. Let $X$ be a finite-dimensional locally compact Hausdorff space and let $R^{t}$ be the euclidean $t$-space. Then

$$
\operatorname{dim}\left(X \times R^{\iota}\right)=(\operatorname{dim} X)+t .
$$

Lemma 6. Let $X$ be a finite-dimensional locally compact Hausdorff space and let $Y$ be a closed subset of $X$. Then

$$
\operatorname{dim} X=\max (\operatorname{dim} Y, \operatorname{dim}(X-Y)) .
$$

Lemma 6 is an immediate consequence of some results of [9].

Lemma 7. Let $A_{\alpha}$ be a collection of open subsets of a non-null finite-dimensional locally compact Hausdorff space $X$ indexed by a well-ordered set $\{\alpha\}$. If $\mathrm{U}_{\alpha} A_{\alpha}=X$ and $\alpha^{\prime}>\alpha$ implies $A_{\alpha^{\prime}} \supset A_{\alpha}$, then there is some index $\beta$ such that

$$
\operatorname{dim} X=\operatorname{dim}\left(A_{\beta}-\bigcup_{\alpha<\beta} A_{\alpha}\right) .
$$

Proof. Let $K$ be a compact subset of $X$ which is of the same dimension as $X$. Since $K \subset X=\cup_{\alpha} A_{\alpha}$, it follows that $K$ is covered by a finite number of the sets $A_{\alpha}$. But $A_{\alpha}$ is ordered by inclusion; we infer that there is some $A_{\gamma}$ which contains $K$ and then is of the same dimension as $X$. Since $A_{\alpha}$ is well- 
ordered, there is a minimal $\beta$ such that $A_{B}$ and $X$ are of the same dimension. Hence the conclusion of our lemma follows from Lemma 6 if we can show that the dimension of $U_{\alpha<\beta} A_{\alpha}$ is less than that of $X$.

If there is an index $\gamma$ preceding to $\beta$, then $\mathrm{U}_{\alpha<\beta} A_{\alpha}=A_{\gamma}$ and hence our assertion follows from the minimality of $\beta$. Suppose now that there is no index preceding to $\beta$. If $\beta$ is the first index, then our assertion is trivial. If $\beta$ is not the first index, then as shown in the preceding paragraph, $\mathrm{U}_{\alpha<\beta} A_{\alpha}$ and $A_{\gamma}$ are of the same dimension for some $\gamma<\beta$. Hence again our assertion follows from the minimality of $\beta$.

Lemma 8. Let $X$ be a finite-dimensional locally compact Hausdorff space of type $P_{n}(\mathfrak{S})$. Then for any bounded open subset $Q$ of $X$ and any integer $k>n$,

$$
H_{k}(X, X-Q ; \mathfrak{E})=0 .
$$

If in particular $\mathfrak{E}=\mathfrak{B}, X$ is of dimension $n$.

Proof. Since $X$ is finite-dimensional, there is a greatest integer $m$ such that for some bounded open subset $Q$ of $X$

$$
H_{m}(X, X-Q ; \mathfrak{S}) \neq 0 .
$$

Let $e$ be a nonzero element of $H_{m}(X, X-Q$; (E) . By Lemma 1 there exists a minimal closed set $M$ contained in $\bar{Q}$ and such that for some $e^{\prime} \in H_{m}(M$ $\cup(X-Q), X-Q ; \mathfrak{s})$,

$$
e=e^{\prime} \mid(X, X-Q) .
$$

Since $e \neq 0, M \cap Q \neq \varnothing$ and then we may take a point $x$ of $M \cap Q$. Suppose that $m>n$; then there exists, by the definition of the property $P_{n}(\mathfrak{E})$, a neighborhood $A$ of $x$ contained in $Q$ and such that

$$
H_{m}(X, X-Q ; \mathfrak{E}) \mid(X, X-A)=0 .
$$

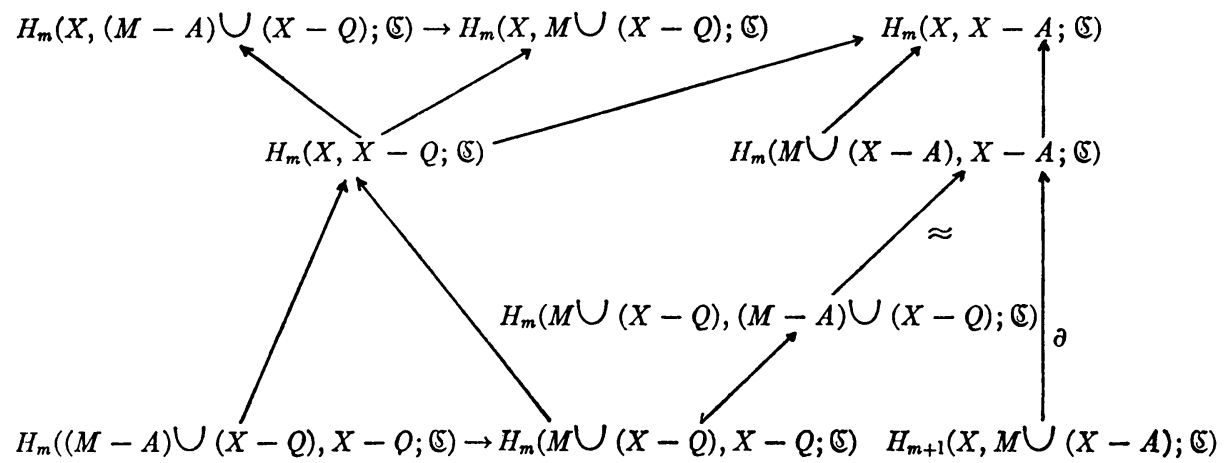

Because of our choice of $M$,

$$
e^{\prime} \notin H_{m}\left((M-A) \cup_{-}(X-Q), X-Q ;(\mathfrak{E}) \mid(M \cup(X-Q), X-Q) ;\right.
$$


therefore $e^{\prime} \mid(M \cup(X-Q),(M-A) \cup(X-Q)) \neq 0$ and hence

$$
e^{\prime} \mid(M \cup(X-A), X-A) \neq 0 .
$$

Since $e^{\prime}|(X, X-A)=e|(X, X-A)=0$, it follows that

$$
e^{\prime} \mid(M \cup(X-A), X-A) \in \partial H_{m+1}(X, M \cup(X-A) ; \text { (5) }) .
$$

Hence $H_{m+1}(X, M \cup(X-A)$; $\mathfrak{E}) \neq 0$, contrary to our assumption on $m$. This proves that $m \leqq n$.

On the other hand, there is a fundamental $(\mathbb{S}, n)$-pair $(U, S)$. For such a bounded open set $U, H_{n}(X, X-U ;(\mathfrak{S}) \neq 0$. Hence $m \geqq n$ and consequently $m=n$.

Suppose now that $\mathfrak{E}=\mathfrak{B}$. If $(U, S)$ is a fundamental $(\mathfrak{P}, n)$-pair, then $H_{n}(\bar{U}, \bar{U}-U ; \mathfrak{B}) \neq 0$ as it is isomorphic to $H_{n}(X, X-U ; \mathfrak{P})$. Hence by Lemma $4, X$ is of dimension $\geqq n$.

For any compact subsets $M, N$ of $X$ with $M \supset N$, and any integer $k>n$, $H_{k}(M, N ; \mathfrak{B})=0$. In fact, let $Q$ be a bounded open subset of $X$ such that $M-Q=N$. Then the composition

$$
H_{k}(M, N ; \mathfrak{P}) \rightarrow H_{k}(M \cup(X-Q), N \cup(X-Q) ; \mathfrak{B}) \rightarrow H_{k}(X, X-Q ; \mathfrak{B})
$$

is an isomorphism into and hence our assertion follows. This proves that $X$ is of dimension $\leqq n$.

Lemma 9. Let $X$ be a finite-dimensional locally compact Hausdorff space of type $P_{n}(\mathfrak{S})$. Let $M, N$ be compact subsets of $X$ with $M \supset N$. If there is a nonzero element $e$ of $H_{n}(M, N$; $\mathfrak{E})$ such that for no proper closed subset $K$ of $(M-N)$, $e \mid(M, K \cup N)=0$, then $M-N$ is open in $X$. Hence if $\mathfrak{C}=\mathfrak{B}$, a closed subset of $X$ is of dimension $n$ if and only if it is somewhere dense.

Proof. Let $x$ be a point of $M-N$ and let $(U, S)$ be a fundamental (ㄷ, $n)$ pair with $U$ containing $x$. Then $x$ has a neighborhood $V$ contained in $U-N$ and such that for some $u \in S, e|(X, X-V)=u|(X, X-V)$. By hypothesis, $e \mid(M, M-V) \neq 0$ and then $e \mid(M \cup(X-V), X-V) \neq 0$. Since

$$
H_{n+1}(X, M \cup(X-V) ; \mathfrak{C})=0
$$

(Lemma 8), it follows that $e \mid(X, X-V) \neq 0$. Hence $u \neq 0$.

Suppose that $V \nsubseteq M$. Then $W=V-M$ is a non-null open subset of $U$. Therefore

On the other hand,

$$
u \mid(X, X-W) \neq 0 .
$$

$$
u|(X, X-W)=e|(X, X-W)=0 .
$$

Hence we have arrived at a contradiction. This proves that $x$ is an inner point of $M-N$. Since $x$ is arbitrary, it follows that $M-N$ is open.

Suppose now that $\mathfrak{E}=\mathfrak{P}$ and let $X^{\prime}$ be a closed subset of $X$. If $X^{\prime}$ is $n$ - 
dimensional, there exist, by Remark 3, compact subsets $M, N$ of $X^{\prime}$ such that $M \supset N$ and for some nonzero element $e$ of $H_{n}(M, N ; \mathfrak{P}), e \mid(M, K \cup N) \neq 0$ whenever $K$ is a proper compact subset of $(M-N)^{-}$. It follows that $M-N$ is open. Hence $X^{\prime}$ is somewhere dense. Conversely, if $X^{\prime}$ is somewhere dense, then the interior of $X^{\prime}$ is not null and therefore is $n$-dimensional. Hence $X^{\prime}$ is $n$-dimensional.

Lemma 10. Let $X$ be a finite-dimensional locally compact Hausdorff space of type $P_{n}(\mathbb{S})$ and let $Q$ be a connected non-null bounded open subset of $X$. Then for any non-null open subset $Q^{\prime}$ of $Q$, the natural homomorphism of

$$
H_{n}(X, X-Q ;()
$$

into $H_{n}\left(X, X-Q^{\prime}\right.$; (S) is one-one. If in particular there is a fundamental

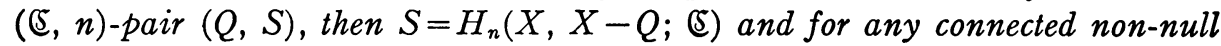
open subset $Q^{\prime}$ of $Q$, the natural homomorphism of $H_{n}(X, X-Q$; (5) into $H_{n}\left(X, X-Q^{\prime}\right.$; (5) is one-one and onto.

Proof. Let $e$ be a nonzero element of $H_{n}(X, X-Q$; (5). By Lemma 1, there is a minimal compact subset $M$ of $\bar{Q}$ such that for some

$$
e^{\prime} \in H_{n}(M \cup(X-Q), X-Q ; \mathfrak{E}), e=e^{\prime} \mid(X, X-Q) .
$$

Clearly there is no proper compact subset $K$ of $(M \cap Q)^{-}$such that

$$
e^{\prime} \mid(M \cup(X-Q), K \cup(X-Q))=0 .
$$

Since the natural homomorphism of $H_{n}(M, M-Q$; $(\mathfrak{C})$ into

$$
H_{n}(M \cup(X-Q), X-Q \text {; } \mathfrak{S})
$$

is one-one and onto, we may apply Lemma 9. Therefore $M \cap Q$ is open in $X$. But $M \cap Q$ is clearly non-null and closed in $Q$. It follows from the connectedness of $Q$ that $M \cap Q=Q$. Hence

$$
M=\bar{Q} .
$$

It is an immediate consequence of the minimality of $M$ that whenever $Q^{\prime}$ is a non-null open subset of $Q$,

$$
e \mid\left(X, X-Q^{\prime}\right) \neq 0 \text {. }
$$

Since $e$ is an arbitrary nonzero element of $H_{n}(X, X-Q$; $(\mathfrak{S})$, the natural homomorphism of $H_{n}\left(X, X-Q\right.$; (א) into $H_{n}\left(X, X-Q^{\prime}\right.$; $\left.\mathfrak{E}\right)$ is one-one.

Suppose that there is a fundamental $(\mathbb{C}, n)$-pair $(Q, S)$. For any connected non-null open subset $Q^{\prime}$ of $Q$ we take a point $x$ of $Q^{\prime}$. 'Then there is a neighborhood $A$ of $x$ contained in $Q^{\prime}$ and such that

$$
H_{n}\left(X, X-Q^{\prime} ; \mathfrak{S}\right)|(X, X-A) \subset S|(X, X-A) .
$$

Since the natural homomorphism of $H_{n}\left(X, X-Q\right.$; (5) into $H_{n}(X, X-A$; (5) 
and that of $H_{n}\left(X, X-Q^{\prime} ;(\mathfrak{S})\right.$ into $H_{n}(X, X-A ;(\mathfrak{S})$ are both one-one, it follows that the natural homomorphism of $H_{n}\left(X, X-Q\right.$; (E) into $H_{n}\left(X, X-Q^{\prime}\right.$; (5) maps $S$ isomorphically onto $H_{n}\left(X, X-Q^{\prime}\right.$; (E). Let $Q^{\prime}=Q$; then we have

$$
S=H_{n}(X, X-Q ; \mathfrak{E}) \text {. }
$$

\section{4. (ㄷ, $n$ )-manifolds.}

Definition. A $(\mathbb{S}, n)$-manifold is a connected, finite-dimensional, locally compact Hausdorff space of type $P_{n}(\mathbb{E})$.

It is easily seen that every $(\mathbb{S}, n)$-manifold is locally connected [7].

REMARK 4. An $n$-dimensional manifold in the ordinary sense is a (ㅌ, $n)$ manifold. The dimension of a $(\mathfrak{C}, n)$-manifold is not less than $n$ and is equal to $n$ if $\mathfrak{E}=\mathfrak{P}$ (Lemma 8).

On a $(\mathfrak{S}, n)$-manifold the fundamental $(\mathfrak{S}, n)$-pairs $(U, S)$ with $U$ connected are of particular importance. As shown in Lemma 10, the group $S$ of such a fundamental $(\mathfrak{E}, n)$-pair $(U, S)$ is equal to $H_{n}(X, X-U$; (5) which is determined by $U$ and is isomorphic to $\mathfrak{E}$. Therefore we may use $(U,[\mathbb{E}])$ to denote such a fundamental (ㅌ, $n)$-pair.

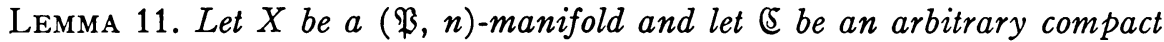
abelian group. If $(U,[\mathfrak{P}])$ is a fundamental $(\mathfrak{B}, n)$-pair with $U$ connected, then there is a fundamental $(\mathfrak{S}, n)$-pair $(U,[\mathfrak{G}])$. Hence every $(\mathfrak{B}, n)$-manifold is a (ㄷ, $n)$-manifold.

Proof. Let $X$ be a $(\mathfrak{B}, n)$-manifold. Whenever $Q$ is a bounded open subset of $X$, we denote by $H^{k}(X, X-Q)$ the $k$ th integral Cech cohomology group of $(X, X-Q)$. It is known [10] that

$$
H_{k}\left(X, X-Q ;(\mathfrak{s})=\operatorname{Hom}\left(H^{k}(X, X-Q) \text {, ( }\right) \oplus \operatorname{Ext}\left(\mathfrak{E}, H^{k+1}(X, X-Q)\right)\right. \text {. }
$$

Let $(U,[\mathfrak{P}])$ be a fundamental $(\mathfrak{P}, n)$-pair with $U$ connected. Since $X$ is of dimension $n$ (Lemma 8 ), $H^{n+1}(X, X-U)=0$. Therefore $H^{n}(X, X-U)$ is isomorphic to the additive group of integers and hence $H_{n}(X, X-U$; (E) is isomorphic to $\mathbb{E}$. Now we claim that $\left(U, H_{n}(X, X-U ; \mathfrak{E})\right)$ is a fundamental $(\mathbb{C}, n)$-pair.

For any connected non-null open subset $W$ of $U$, the natural homomorphism of $H_{n}(X, X-U ; \mathfrak{B})$ into $H_{n}(X, X-W ; \mathfrak{B})$ is one-one and onto (Lemma 10) and then so is the natural homomorphism of $H^{n}(X, X-W)$ into $H^{n}(X, X-U)$. Therefore the natural homomorphism of $H_{n}(X, X-U$; (5) into $H_{n}(X, X-W ;(\mathfrak{S})$ is one-one and onto. Hence for any non-null open subset $W$ of $U$ the natural homomorphism of $H_{n}\left(X, X-U\right.$; (E) into $H_{n}(X, X-W$; (E) is one-one.

Let $y$ be a point of $U$ and let $V$ be a neighborhood of $y$. Then there is a neighborhood $W$ of $y$ contained in $U \cap V$ and such that $H_{n}(X, X-V ; \mathfrak{B})$ $\left|(X, X-W) \subset H_{n}(X, X-U ; \mathfrak{B})\right|(X, X-W)$ and for $k \neq n, H_{k}(X, X-V ; \mathfrak{B})$ $(X, X-W)=0$. Since $X$ is locally connected, we may assume $W$ connected. 
Therefore $H_{n}\left(X, X-U\right.$; (5) $\mid(X, X-W)=H_{n}(X, X-W$; (5) (Lemma 10). Hence

$$
H_{n}\left(X, X-V ;(\mathfrak{E})\left|(X, X-W) \subset H_{n}(X, X-U ; \mathfrak{E})\right|(X, X-W) .\right.
$$

Since $H^{n}(X, X-W)$ is isomorphic to the additive group of integers, Ext $\left(\mathfrak{S}, H^{n}(X, X-W)\right)=0$. Therefore the natural homomorphism of Ext $\left(\mathbb{E}, H^{n}(X, X-V)\right)$ into $\operatorname{Ext}\left(\mathfrak{S}, H^{n}(X, X-W)\right)$ is trivial. Let $k \neq n$. Since $H_{k}(X, X-V ; \mathfrak{P}) \mid(X, X-W)=0$, the natural homomorphism of $H^{k}(X, X-W)$ into $H^{k}(X, X-V)$ is trivial. Therefore the homomorphism of

$$
\text { Hom }\left(H^{k}(X, X-V), \text { (5) into Hom }\left(H^{k}(X, X-W), \mathfrak{s}\right)\right.
$$

and that of Ext $\left(\mathfrak{E}, H^{k}(X, X-V)\right)$ into Ext $\left(\mathfrak{S}, H^{k}(X, X-W)\right)$ are both trivial. Hence for $k \neq n$,

$$
H_{k}(X, X-V ; \mathfrak{S}) \mid(X, X-W)=0 .
$$

5. $\mathfrak{E}$-orientability. Let $X$ be a $(\mathfrak{S}, n)$-manifold and let $\mathfrak{F}$ be the totality of fundamental $(\mathfrak{S}, n)$-pairs $(U,[\mathfrak{E}])$ with $U$ connected. An ordered pair of elements $(U,[\mathbb{E}]),(V,[\mathfrak{E}])$ of $\mathfrak{F}$ is called a step if either $U \subset V$ or $V \subset U$. A finite sequence of elements of $\mathfrak{F}$

$$
\left(U_{1},[\mathfrak{s}]\right), \cdots,\left(U_{t},[\mathfrak{s}]\right)
$$

is called a path from $\left(U_{1},[\mathfrak{⿷}]\right)$ to $\left(U_{t},[\mathfrak{\Im}]\right)$ if every pair of adjacent terms is a step. For each $i=1, \cdots, t-1$, we have either $U_{i} \subset U_{i+1}$ or $U_{i} \supset U_{i+1}$; it follows from Lemma 10 that there is a natural isomorphism of $H_{n}\left(X, X-U_{i}\right.$; (5) onto $H_{n}\left(X, X-U_{i+1} ;(\mathbb{E})\right.$. The composition of these isomorphisms is an isomorphism of $H_{n}\left(X, X-U_{1}\right.$; (夭) onto $H_{n}\left(X, X-U_{t}\right.$; ( $)$, called the isomorphism induced by the path.

Definition. A $(\mathfrak{}, n)$-manifold is $(\mathfrak{S}$-orientable if there is a fundamental $(\mathfrak{S}, n)$-pair $(U,[\mathbb{G}])$ with $U$ connected and such that every path from $(U,[\mathfrak{G}])$ to itself induces the identity isomorphism on $H_{n}(X, X-U$; (্)).

REMARK 5. The following are equivalent.

(i) $X$ is (5-orientable.

(ii) For every fundamental (, $n)$-pair $(U,[\mathbb{E}])$ with $U$ connected, every path from $(U,[\mathbb{E}])$ to itself induces the identity isomorphism on $H_{n}(X$, $X-U$; (5).

(iii) There exist two fundamental $(\mathfrak{E}, n)$-pairs $(U,[\mathfrak{G}])$ and $(V,[\mathfrak{G}])$ such that $U$ and $V$ are connected and all the paths from $(U,[\mathbb{E}])$ to $(V,[\mathbb{E}])$ induces the same isomorphism of $H_{n}(X, X-U$; $\mathfrak{E})$ onto $H_{n}(X, X-V$; $\mathfrak{E})$.

(iv) The statement (iii) holds for any two fundamental $(\mathcal{S}, n)$-pairs ( $U,[\mathfrak{S}]),(V,[\mathfrak{S}])$ with $U, V$ connected.

REMARK 6. Every point of a $(\mathfrak{E}, n)$-manifold has a $(\mathfrak{S}$-orientable neighborhood.

REMARK 7. An orientable $n$-dimensional manifold in the ordinary sense

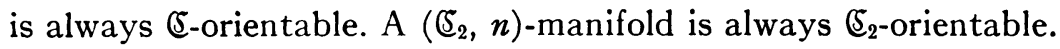




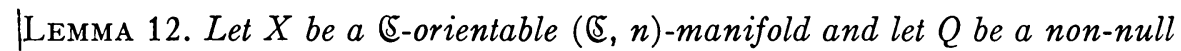
bounded open subset of $X$. Then there is a subgroup $S$ of $H_{n}(X, X-Q$; (5) such that $(Q, S)$ is a fundamental ( $(\mathfrak{S}, n)$-pair.

Proof. We first prove that, if $Q$ is connected, then $\left(Q, H_{n}(X, X-Q ;\right.$ ( $\left.)\right)$ is a fundamental $(\mathfrak{E}, n)$-pair.

For each point $x$ of $\bar{Q}$ there is a fundamental $(\mathbb{E}, n)$-pair ( $U$, [] $]$ ) with $U$ connected and containing $x$. Since $\bar{Q}$ is compact, there exist a finite number of fundamental $(\mathfrak{C}, n)$-pairs

$$
\left(U_{1},[\mathfrak{飞}]\right),\left(U_{2},[\mathfrak{飞}]\right), \cdots,\left(U_{t},[\mathfrak{飞}]\right)
$$

such that each $U_{i}$ is connected and $U_{1} \cup \ldots \cup U_{t} \supset \bar{Q}$. Since $\bar{Q}$ is connected, we may assume that for each $i=1, \cdots, t-1$,

$$
\left(U_{1} \cup \ldots \cup U_{i}\right) \cap U_{i+1} \neq 0 .
$$

Since $X$ is $\left(\mathfrak{E}\right.$-orientable, all the paths from $\left(U_{1},[\mathfrak{E}]\right)$ to $\left(U_{2},[\mathfrak{E}]\right)$ induces the same isomorphism $h$ of $H_{n}\left(X, X-U_{1}\right.$; (א) onto $H_{n}\left(X, X-U_{2}\right.$; $\left.\mathfrak{S}\right)$. Therefore whenever $e \in H_{n}\left(X, X-U_{1}\right.$; $(\mathfrak{E})$,

$$
e|(X, X-V)=h(e)|(X, X-V)
$$

for all components $V$ of $U_{1} \cap U_{2}$ and so

$$
e\left|\left(X, X-\left(U_{1} \cap U_{2}\right)\right)-h(e)\right|\left(X, X-\left(U_{1} \cap U_{2}\right)\right)=0 .
$$

By the exactness of the Mayer-Vietoris sequence of the triad $\left(X ; X-U_{1}\right.$, $\left.X-U_{2}\right)$, there is some $e^{\prime} \in H_{n}\left(X, X-\left(U_{1} \cup U_{2}\right)\right.$; (S) such that

$$
e^{\prime}\left|\left(X, X-U_{1}\right)=e, \quad e^{\prime}\right|\left(X, X-U_{2}\right)=h(e) .
$$

Since $U_{1} \cup U_{2}$ is connected, it follows from Lemma 10 that the natural homomorphism of $H_{n}\left(X, X-\left(U_{1} \cup U_{2}\right)\right.$; (5) into $H_{n}\left(X, X-U_{1}\right.$; (5) is one-one. Hence it is an isomorphism onto. This isomorphism followed by $h$ is the natural homomorphism of $H_{n}\left(X, X-\left(U_{1} \cup U_{2}\right)\right.$; (5) $)$ into $H_{n}\left(X, X-U_{2}\right.$; (5) which is also an isomorphism onto. Then we can easily see that $\left(U_{1} \cup U_{2}\right.$, $H_{n}\left(X, X-\left(U_{1} \cup U_{2}\right)\right.$; () ) is a fundamental (હ, $\left.n\right)$-pair.

Repeating this process, we can finally have a fundamental ( $(5, n)$-pair $\left(U_{1} \cup \ldots \cup U_{t}, H_{n}\left(X, X-\left(U_{1} \cup \ldots \cup U_{t}\right) ;(\mathbb{S})\right)\right.$. Hence $\left(Q, H_{n}(X, X-Q\right.$; $(\mathfrak{S}))$ is a fundamental $(\mathfrak{S}, n)$-pair.

Now let $Q$ be any non-null bounded open subset of $X$. Since $X$ is connected, there is a connected bounded open subset $Q^{\prime}$ of $X$ containing $Q$. We

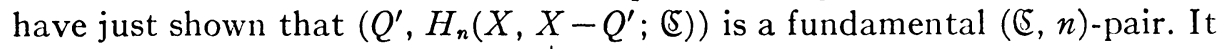

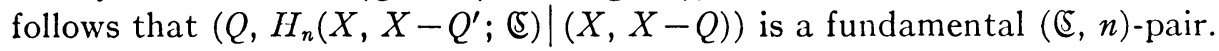

CoRollary. For any connected non-null bounded open subset $U$ of a (5orientable (ㅌ, n)-manifold there is a fundamental (ㅌ, $n)$-pair $(U,[\mathfrak{S}])$. Hence

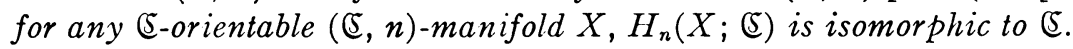




\section{Local separation.}

Lemma 13. Let $X$ be a $(\mathfrak{S}, n)$-manifold and let $M, N$ be compact subsets of $X$ with $M \supset N$. If there is a nonzero element e of $H_{n-1}(M, N$; $\mathfrak{E})$ such that for no proper compact subset $K$ of $(M-N)^{-}, e \mid(M, K \cup N)=0$, then every point $x$ of $M-N$ has a neighborhood $Q$ such that every neighborhood of $x$ meets at least two components of $Q-M$.

Proof. Let $x$ be a point of $M-N$ and let $(U,[\mathfrak{\complement}])$ be a fundamental $(\mathfrak{E}, n)$ pair such that $U$ is connected and $x \in U \subset X-N$. Let $Q$ be a neighborhood of $x$ contained in $U$ and such that

$$
H_{n-1}(X, X-U ; \mathfrak{E}) \mid(X, X-Q)=0 .
$$

The closure of $Q-M$ contains $Q$. Otherwise, $Q^{\prime}=Q-(Q-M)^{-}$is a nonnull open set contained in $M$. It follows from

$$
\begin{aligned}
& H_{n-1}\left(M, N \text {; () } \rightarrow H_{n-1}(X, X-U \text {; } \mathfrak{S}) \rightarrow H_{n-1}(X, X-Q \text {; } \mathfrak{E})\right. \\
& H_{n-1}\left(M, M-Q^{\prime} ;(\mathfrak{\Im}) \longrightarrow H_{n-1}\left(X, X-Q^{\prime} ; \mathfrak{(}\right)\right.
\end{aligned}
$$

that $e \mid\left(M, M-Q^{\prime}\right)=0$, contrary to our hypothesis.

Suppose that there is a neighborhood of $x$ which meets only one component $A$ of $Q-M$. Then

$$
V=Q-[Q-(M \cup A)]^{-}
$$

is a neighborhood of $x$. Both $V$ and $V-M$ contain $A$ and are contained in $\bar{A}$; therefore they are connected and hence the natural homomorphism of $H_{n}(X, X-V ; \mathfrak{E})$ into $H_{n}(X, M \cup(X-V) \mathfrak{E})$; is one-one and onto.

$$
H_{n-1}\left(X, X-V ;(\mathfrak{c}) \leftarrow H_{n-1}\left(M \cup(X-V), X-V ;(\mathfrak{c}) \stackrel{\partial}{\leftarrow} H_{n}(X, M \cup(X-V) ;(\mathfrak{l})\right.\right.
$$

By hypothesis, $e \mid(M, M-V) \neq 0$. Therefore $e \mid(X, X-V) \neq 0$. On the other hand,

$$
e|(X, X-V)=(e \mid(X, X-U))|(X, X-V)=0 .
$$

Hence we have arrived at a contradiction.

Lemma 14. Let $X$ be a $(\mathfrak{B}, n)$-manifold and let $Y$ be a closed subset of $X$. Then $Y$ is $(n-1)$-dimensional if and only if $Y$ is nowhere dense and there is a point $x$ of $Y$ and a neighborhood $Q$ of $x$ such that every neighborhood of $x$ meets at least two components of $Q-Y$.

Proof. Suppose that $Y$ is $(n-1)$-dimensional; then it is nowhere dense by 
Lemma 9. By Remark 3, there exist compact subsets $M, N$ of $Y$ such that $M \supset N$ and for some nonzero element $e$ of $H_{n-1}(M, N ; \mathfrak{B}), e \mid(M, K \cup N) \neq 0$ whenever $K$ is a proper compact subset of $[M-N]-$. It follows from Lemma 13 that there is a point $x$ of $M-N$ and a neighborhood $Q$ of $x$ such that every neighborhood of $x$ meets at least two components of $Q-M$. Since $Y$ is nowhere dense, no open set can be contained in $Y$. Hence every neighborhood of $x$ meets at least two components of $Q-Y$.

Conversely, suppose that $Y$ is nowhere dense and that there is a point $x$ of $Y$ and a neighborhood $Q$ of $x$ such that every neighborhood of $x$ meets at least two components of $Q-Y$. By Lemma 9, the dimension of $Y$ is $\leqq n-1$. Since $X$ is a $(\mathfrak{B}, n)$-manifold, there is a fundamental $(\mathfrak{B}, n)$-pair $(U,[\mathfrak{P}])$ with $U$ connected and contained in $Q$. Clearly $U-Y$ contains at least two components. Let $A$ be a component of $U-Y$. Let

$$
M=[(\bar{A}-A) \cap U]^{-}, \quad N=M-U .
$$

We can easily see that $M, N$ are compact subsets of $Y$ and that the natural homomorphism of $H_{n-1}(M, N ; \mathfrak{B})$ into $H_{n-1}(\bar{A}-A, \bar{A}-U ; \mathfrak{B})$ is one-one and onto. Since $H_{n}(\bar{A}, \bar{A}-U ; \mathfrak{B})=0$ and $H_{n}(\bar{A}, \bar{A}-A ; \mathfrak{B})$ is isomorphic to $\mathfrak{B}$ (Lemma 10), it follows from

$$
\begin{aligned}
H_{n-1}(M, N ; \mathfrak{B}) & \stackrel{\approx}{\longrightarrow} H_{n-1}(\bar{A}-A, \bar{A}-U ; \mathfrak{B}) \stackrel{\partial}{\longleftarrow} H_{n}(\bar{A}, \bar{A}-A ; \mathfrak{B}) \\
& \longleftarrow H_{n}(\bar{A}, \bar{A}-U ; \mathfrak{B})
\end{aligned}
$$

that $H_{n-1}(M, N ; \mathfrak{B}) \neq 0$. Hence the dimension of $Y$ is $\geqq n-1$ by Lemma 4 . This proves that $Y$ is $(n-1)$-dimensional.

Lemma 15. Let $X$ be a $\left(\mathfrak{S}_{q}, n\right)$-manifold and let $T$ be a periodical transformation on $X$ of period $q$, where $q$ is any prime number. Then the fixed point set $L$ of $T$ is closed (and hence locally compact), locally connected, nowhere dense and of type $P\left(\mathfrak{S}_{q}\right)$. Hence every component of $L$ is open in $L$ and is $a\left(\mathfrak{S}_{q}, k\right)$-manifold, where $k$ depends on the component and is $\leqq n-1$. The equality holds only if $q=2$.

REMARK 8. This statement, though slightly general, is essentially the same as the one given in [5]. Firstly, the conclusion is a local property; therefore the compactness of $X$ required in [5] can be replaced by the local compactness. Secondly, the assumption that every bounded open set is a countable union of compact sets is not essential and then can be omitted. Finally, the property $Q$ used in [5] can be removed, as we can prove that a locally compact Hausdorff space which possesses the property $P_{n}(\mathbb{E})$ at point $x$ possesses also the property $Q(\mathcal{E})$ at $x$ (see the Appendix).

Lemma 16. Let $X$ be a $\left(\mathfrak{\mho}_{2}, n\right)$-manifold, let $T$ be a periodical transformation on $X$ of period 2 and let $L$ be the fixed point set of $T$. If $x$ is a point of $L$ at which $L$ possesses the property $P_{n-1}\left(\mathfrak{S}_{2}\right)($ Lemma 15$)$, then $x$ has a connected neighborhood $Q$ such that $Q=T(Q)=\operatorname{Int} \bar{Q}$ (i.e., the interior of $\bar{Q}$ ) and $Q-L$ has exactly two components which are mapped into each other by $T$. 
Proof. By hypothesis, there is a fundamental $\left(\mathfrak{夭}_{2}, n-1\right)$-pair $(U, S)$ of $L$ with $U$ containing $x$. Since the natural homomorphism of $H_{n-1}\left(\bar{U}, \bar{U}-U\right.$; $\left.\mathfrak{G}_{2}\right)$ into $H_{n-1}\left(L, L-U ; \mathfrak{夭}_{2}\right)$ is one-one and onto, there is an element $e$ of $H_{n-1}\left(\bar{U}, \bar{U}-U ; \mathfrak{E}_{2}\right)$ such that for no proper compact subset $K$ of $\bar{U}$, $e \mid(\bar{U}, K \cup(\bar{U}-U))=0$. It follows from Lemma 13 that there is a neighborhood $A$ of $x$ such that every neighborhood of $x$ meets at least two components of $A-\bar{U}$. Since $L$ is nowhere dense (Lemma 15), every neighborhood of $x$ meets at least two components of $A-L$. Let $B$ be the component of $A \cap T(A)$ containing $x$ and let

$$
Q=\operatorname{Int} \bar{B} \text {. }
$$

It is clear that $Q$ is connected, $Q=T(Q)=\operatorname{Int} \bar{Q}$ and $Q-L$ has at least two components. We remain to prove that the components of $Q-L$ are two in number and are mapped into each other by $T$.

Let $C$ be a component of $Q-L$. Then $T(C) \neq C$, since otherwise, the periodical transformation $T^{\prime}: Q \rightarrow Q$ defined by

$$
T^{\prime}(x)=\left\{\begin{array}{cl}
T(x) & \text { if } x \in C, \\
x & \text { if } x \in Q-C
\end{array}\right.
$$

has a fixed point set which is not nowhere dense, contrary to Lemma 15 .

Now we claim that every point of $Q \cap \bar{C}$ is contained in Int $(\bar{C} \cup T(\bar{C}))$. Let $y$ be a point of $Q \cap \bar{C}$ and let $D$ be a neighborhood of $y$ whose closure is contained in $Q$. In the diagram

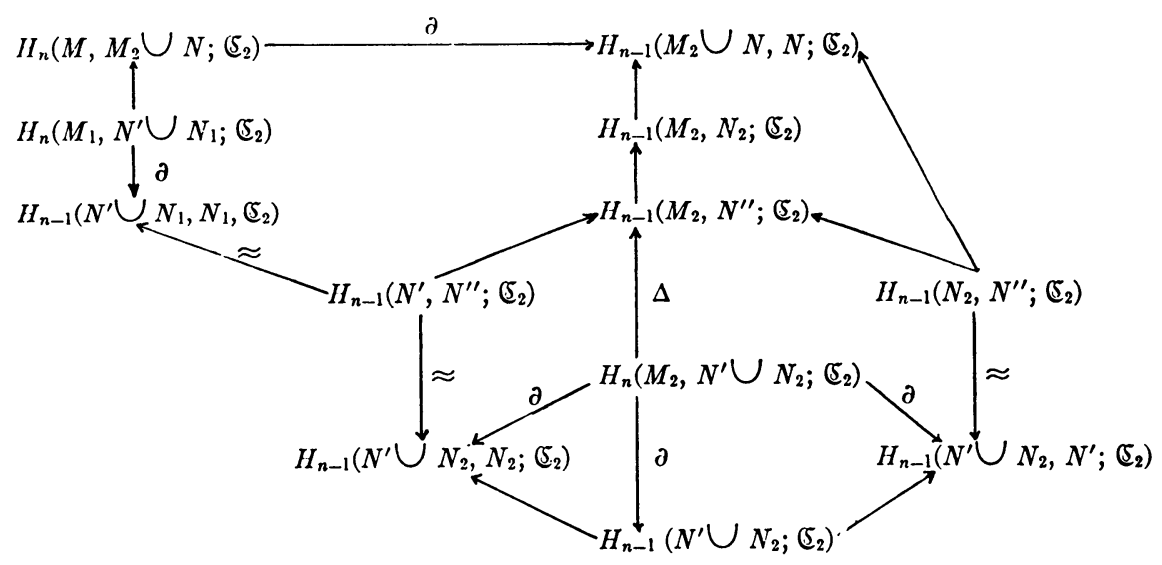

we let

$$
\begin{aligned}
M_{1} & =(C \cap D)^{-}, \quad M_{2}=T\left(M_{1}\right), \quad M=M_{1} \cup M_{2} ; \\
N_{1} & =M_{1}-D, \quad N_{2}=T\left(N_{1}\right), \quad N=N_{1} \cup N_{2} ; \\
N^{\prime} & =M_{1}-C=M_{1} \cap M_{2} ; \\
N^{\prime \prime} & =M_{1}-(C \cup D)=N_{1} \cap N_{2}=N^{\prime} \cap N_{1}=N^{\prime} \cap N_{2} .
\end{aligned}
$$


Since $H_{n}\left(X, X-Q ; \mathfrak{C}_{2}\right)$ is isomorphic to $\mathfrak{C}_{2}$ (Lemmas 10 and 12), there is only one element $u$ of $H_{n}\left(X, X-Q ; \mathfrak{夭}_{2}\right)$ different from 0 . Under the natural homomorphisms

$$
\begin{aligned}
H_{n}\left(X, X-Q ; \mathfrak{\Im}_{2}\right) & \longrightarrow H_{n}\left(X, X-\left(M_{i}-\left(N^{\prime} \cup N_{i}\right)\right) ; \mathfrak{\Im}_{2}\right) \\
& \approx H_{n}\left(M_{i}, N^{\prime} \cup N_{i} ; \mathfrak{夭}_{2}\right)
\end{aligned}
$$

$u\left|\left(X, X-\left(M_{i}-\left(N^{\prime} \cup N_{i}\right)\right)\right)=u_{i}\right|\left(X, X-\left(M_{i}-\left(N^{\prime} \cup N_{i}\right)\right)\right)$ determines a unique nonzero element $u_{i}$ of $H_{n}\left(M_{i}, N^{\prime} \cup N_{i} ;\left(\mathfrak{C}_{2}\right), i=1,2\right.$.

Every group of the above diagram has an element which is corresponding to either $u_{1}$ or $u_{2}$ or both. The one in $H_{n-1}\left(M_{2} \cup N, N ; \mathfrak{\Im}_{2}\right)$ is 0 as

$$
H_{n-1}\left(N_{2}, N^{\prime \prime} ; \mathfrak{S}_{2}\right) \mid\left(M_{2} \cup N, N\right)=0 .
$$

It follows that for some $e \in H_{n}\left(M, N ; \mathfrak{S}_{2}\right)$,

$$
u_{1}\left|\left(M, M_{2} \cup N\right)=e\right|\left(M, M_{2} \cup N\right) .
$$

Let $K$ be a minimal compact subset of $M$ such that for some $e^{\prime} \in H_{n}(K \cup N$, $N ;\left(\mathfrak{S}_{2}\right)$,

$$
e=e^{\prime} \mid(M, N) .
$$

Let $D^{\prime}$ be any neighborhood of $y$ not intersecting $N$. It follows from

$$
\begin{aligned}
e^{\prime} \mid\left(X, X-\left(C \cap D^{\prime}\right)\right) & =e \mid\left(X, X-\left(C \cap D^{\prime}\right)\right) \\
& =u_{1}\left|\left(X, X-\left(C \cap D^{\prime}\right)\right)=u\right|\left(X, X-\left(C \cap D^{\prime}\right)\right) \neq 0
\end{aligned}
$$

that $y \in K$. By Lemma $9, K-N$ is a neighborhood of $y$ contained in $M$. Hence $y$ is an inner point of $\bar{C} \cup T(\bar{C})$.

As a consequence of this result, we have

$$
Q=\operatorname{Int}(\bar{C} \cup T(\bar{C})) \text {. }
$$

In fact, it follows from $Q=\operatorname{Int} \bar{Q}$ that $Q \supset \operatorname{Int}(\bar{C} \cup T(\bar{C}))$. Suppose that our assertion is false. Then there exists, by the connectedness of $Q$, a point of $Q \cap(\bar{C} \cap T(\bar{C}))$ which does not belong to Int $(\bar{C} \cup T(\bar{C}))$. But such a point clearly belongs to $Q \cap \bar{C}$ and then belongs to Int $(\bar{C} \cup T(\bar{C}))$ by our result above. Hence we have arrived at a contradiction. This proves that $Q-L$ has exactly two components $C$ and $T(C)$.

Corollary. Let $X$ be a $(\mathfrak{P}, n)$-manifold, let $T$ be a periodical transformation on $X$ of period 2 and let $L$ be a component of the fixed point set of $T$. If $L$ is a $\left(\mathfrak{G}_{2}, n-1\right)$-manifold (see Lemmas 11 and 15), then $L$ is also a $(\mathfrak{P}, n-1)$-manifold.

Proof. Let $x$ be a point of $L$ and let $(U,[\mathfrak{B}]$ ) be a fundamental (,$n$ )-pair with $U$ connected and containing $x$. Since $X$ is also a $\left(\mathfrak{C}_{2}, n\right)$-manifold (Lemma 11), it follows from Lemma 16 that $x$ has a connected neighborhood 
$V$ such that $V \subset U, T(V)=V$ and $V-L$ has exactly two components $C$ and $T(C) .\left(V, H_{n}(X, X-V ; \mathfrak{B})\right)$ is clearly a fundamental $(\mathfrak{B}, n)$-pair.

For any point $y$ of $L \cap V$ and any neighborhood $Q$ of $y$ there exists, by Lemma 16 and the definition of the property $P_{n}(\mathfrak{B})$, a connected neighborhood $W$ of $y$ such that (i) $W \subset V \cap Q$, (ii) $T(W)=W$, (iii) $W-L$ has exactly two components $D$ and $T(D)$ with $D$ contained in $C$, (iv) the natural homomorphism of $H_{n}(X, X-V ; \mathfrak{B})$ into $H_{n}(X, X-W ; \mathfrak{B})$ is one-one and onto, and (v) whenever $k \neq n$,

$$
H_{k}(X, X-V ; \mathfrak{B}) \mid(X, X-W)=0 .
$$

Now let us observe the natural homomorphism of the Mayer-Vietoris sequence of $(X ; X-C, X-T(C))$ into that of $(X ; X-D, X-T(D))$.

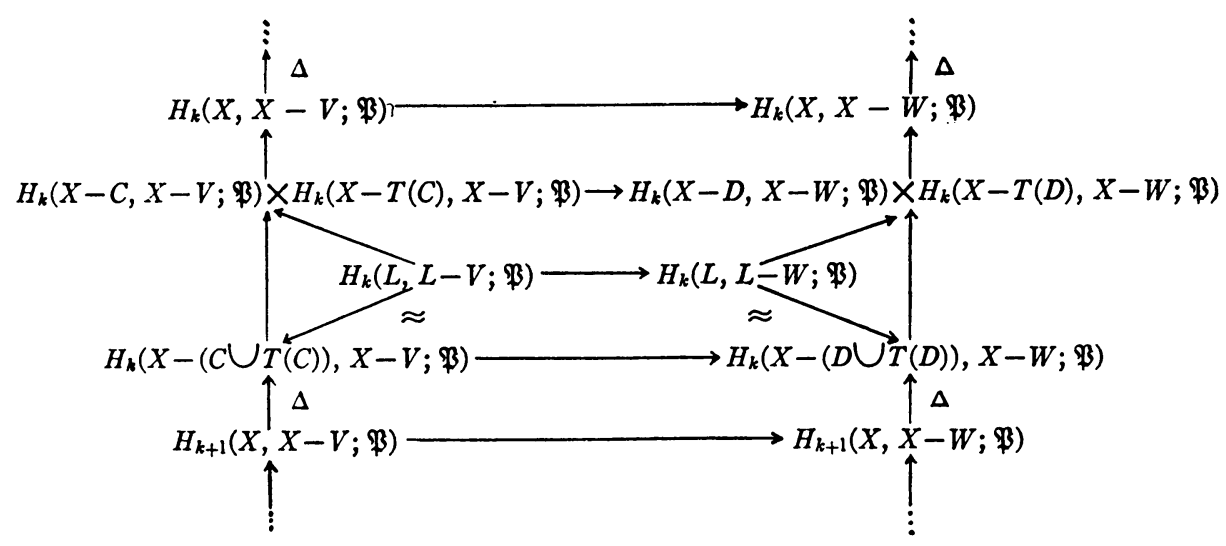

For $k<n$, the natural homomorphism

$$
\begin{aligned}
H_{k}(X-C, X & -V ; \mathfrak{P}) \times H_{k}(X-T(C), X-V ; \mathfrak{P}) \\
& \rightarrow H_{k}(X-D, X-W ; \mathfrak{B}) \times H_{k}(X-T(D), X-W ; \mathfrak{P})
\end{aligned}
$$

is trivial. In fact, let $e$ be any element of $H_{k}(X-C, X-V ; \mathfrak{P})$. The image of $(e \mid(X-D, X-W), 0)$ in $H_{k}(X, X-W ; \mathfrak{B})$ is 0 ; therefore $(e \mid(X-D, X-W)$, $0)$ is the image of some $e^{\prime} \in H_{k}(X-(D \cup T(D)), X-W$; $\mathfrak{B})$. Since $e^{\prime}$ is invariant under the homomorphism induced by $T$, it follows that the isomorphism of $H_{k}(X-D, X-W ; \mathfrak{B})$ onto $H_{k}(X-T(D), X-W$; $\mathfrak{P})$, induced by $T$, maps $e \mid(X-D, X-W)$ into 0. Hence $e \mid(X-D, X-W)=0$.

Let $A$ be a neighborhood of $y$ contained in $W$ and such that whenever $k<n-1$,

$$
H_{k+1}(X, X-W ; \mathfrak{B}) \mid(X, X-A)=0 .
$$

If $k<n-1$ and $e \in H_{k}(L, L-V$; $\mathfrak{B})$, then 


$$
e \mid(X-(D \cup T(D)), X-W)=\Delta e^{\prime}
$$

for some $e^{\prime} \in H_{k+1}(X, X-W ; \mathfrak{B})$. It follows from $e^{\prime} \mid(X, X-A)=0$ that $e \mid(L, L-A)=0$. This proves that whenever $k<n-1$,

$$
H_{k}(L, L-V ; \mathfrak{B}) \mid(L, L-A)=0 .
$$

Since $L$ is of dimension $\leqq n-1$, it follows that whenever $k>n-1$, $H_{k}(L, L-V ; \mathfrak{P})=0$ and hence

$$
H_{k}(L, L-V ; \mathfrak{B}) \mid(L, L-A)=0 .
$$

It is clear that the natural homomorphism

$$
H_{n}(X-C, X-V ; \mathfrak{B}) \times H_{n}(X-T(C), X-V ; \mathfrak{B}) \rightarrow H_{n}(X, X-V ; \mathfrak{B})
$$

is trivial. Therefore there is a subgroup $S$ of $H_{n-1}(L, L-V ; \mathfrak{B})$ such that $\Delta$ maps $H_{n}(X, X-V ; \mathfrak{B})$ isomorphically onto $S \mid(X-(C \cup T(C)), X-V)$. Similarly there is a subgroup $S^{\prime}$ of $H_{n}(X, X-W$; $\mathfrak{B})$ isomorphically $S^{\prime} \mid(X-(D \cup T(D)), X-W)$. Therefore it is clear that the natural homomorphism of $H_{n-1}(L, L-V ; \mathfrak{B})$ in to $H_{n-1}(L, L-W$; $)$ maps $S$ isomorphically onto $S^{\prime}$. For any $e \in H_{n-1}(L, L-V ; \mathfrak{B})$, the image of $e \mid(L, L-W)$ in $H_{n-1}(X-D, X-W ; \mathfrak{B}) \times H_{n-1}(X-T(D), X-W ; \mathfrak{B})$ is 0 ; therefore $e \mid(X-(D \cup T(D)), X-W)$ belongs to $\Delta H_{n}(X, X-W ; \mathfrak{B})$. Hence e $(L, L-W) \in S^{\prime}$.

From this result it follows that

$$
H_{n-1}(L, L-V ; \mathfrak{B})|(L, L-A)=S|(L, L-A) .
$$

With $W, A$ in place of $V, Q$ we can find a neighborhood $W^{\prime}$ of $y$ in $W$. Therefore the natural homomorphism of $H_{n-1}(L, L-W ; \mathfrak{P})$ into $H_{n-1}\left(L, L-W^{\prime} ; \mathfrak{B}\right)$ maps $S^{\prime}$ isomorphically into $H_{n-1}\left(L, L-W^{\prime} ; \mathfrak{B}\right)$. Hence the natural homomorphism of $H_{n-1}(L, L-V ; \mathfrak{B})$ into $H_{n-1}(L, L-A ; \mathfrak{B})$ maps $S$ isomorphically onto $S \mid(L, L-A)$.

Combining all these results, one can easily see that $(L \cap V, S)$ is a fundamental $(\mathfrak{B}, n-1)$-pair. This proves that $L$ possesses that property $P_{n-1}(\mathfrak{B})$ at $x$. But $x$ is an arbitrary point of $L$; hence $L$ is a ( $(n-1)$-manifold.

REMARK 9. From this Corollary the following general question is raised. If $X$ is a $(\mathfrak{B}, n)$-manifold and $T$ is a periodical transformation on $X$ of prime period, is every component of the fixed point set of $T$ a homological manifold with respect to $\mathfrak{B}$ ?

\section{Theorem 1.}

THEOREM 1. Let $X$ be a ( $\mathfrak{B}, n)$-manifold and let $H$ be a finite group acting effectively on $X$. If $H$ is not trivial, the dimension of the fixed point set of $H$ is $\leqq n-1$. The equality holds only if the order of $H$ is 2 .

Proof. Let $T$ be an element of $H$ of prime order $q$. Let $L$ be the fixed point set of $T$ and let $L^{\prime}$ be the fixed point set of $H$. 
Since a $(\mathfrak{B}, n)$-manifold is of dimension $n, L$ is of dimension $\leqq n$. By Lemma $11, X$ is also a $\left(\mathfrak{S}_{q}, n\right)$-manifold; it follows from Lemmas 15 and 9 that $L$ is of dimension $\leqq n-1$.

Since $L^{\prime} \subset L$, the dimension of $L^{\prime}$ is $\leqq n-1$. Now we assume that $L^{\prime}$ is of dimension $n-1$; then $L$ is also of dimension $n-1$.

By Lemma 14, there is a point $x$ of $L^{\prime}$ and a neighborhood $Q$ of $x$ such that every neighborhood of $x$ meets at least two components of $Q-L$.

Let $(U, S)$ be a fundamental $(\mathfrak{B}, n)$-pair with $U$ containing $x$. Since both $X$ and $L$ are locally connected, there is a connected neighborhood $V$ of $x$ contained in $Q \cap U$ and such that $L \cap V$ is connected. It is clear that $(V,[\mathfrak{B}])$ is a fundamental $(\mathfrak{B}, n)$-pair and that $V-L$ has at least two components.

Let $B$ be a component of $V-L$. Then $(B,[\mathfrak{P}])$ is a fundamental $(\mathfrak{P}, n)$ pair. By Lemma $11,\left(B,\left[\mathfrak{E}_{q}\right]\right)$ is a fundamental $\left(\mathfrak{E}_{q}, n\right)$-pair; therefore $H_{n}\left(\bar{B}, \bar{B}-B ; \mathbb{S}_{q}\right)$ has a nonzero element $u$. Let $M=\bar{B}-B$; then $V \cap M$ is not null and is contained in $L$. Let $y$ be a point of $V \cap M$ and let $N=(V \cap M)^{-}$. For any neighborhood $A$ of $y$ contained in $V$ there is a unique element $e_{A}$ of $H_{n-1}\left(N, N-A ; \mathfrak{\complement}_{q}\right)$ such that

$$
e_{A} \mid(M, M-A)=\partial u,
$$

where $\partial$ is the boundary operator of the homology sequence of the triple $(\bar{B}, M, M-A)$.

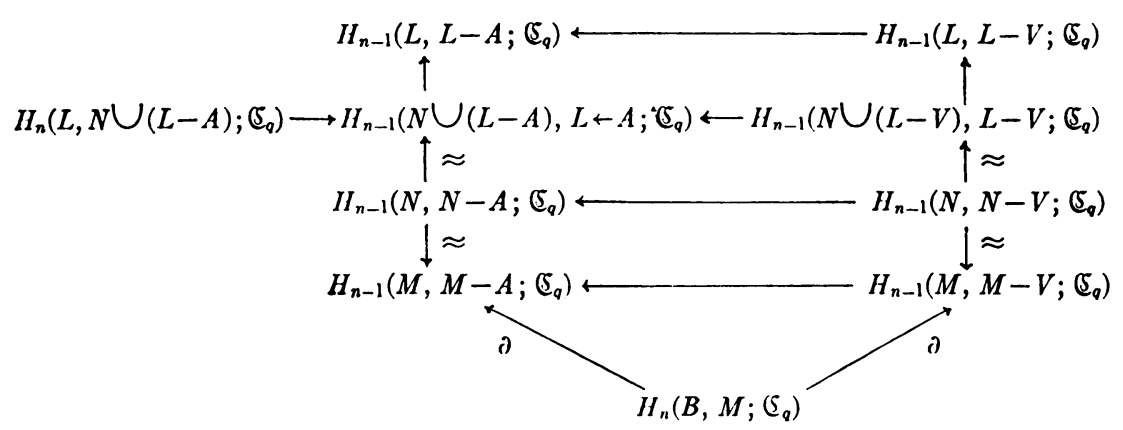

Since the dimension of $L$ is $n-1, H_{n}\left(L, N \cup(L-A)\right.$; $\left.\mathfrak{C}_{q}\right)=0$. Therefore

$$
e_{A}^{\prime}=e_{A} \mid(L, L-A) \neq 0 .
$$

Now $e_{V}^{\prime}$ is an element of $H_{n-1}\left(L, L-V ; \mathbb{S}_{q}\right)$ such that for every neighborhood $A$ of $y$ contained in $V$,

$$
e_{V}^{\prime} \mid(L, L-A)=e_{A}^{\prime} \neq 0 .
$$

It follows that $L$ possesses the property $P_{n-1}\left(\aleph_{q}\right)$ at $y$ (Lemma 15). Since $L \cap V$ is connected and contains both $x$ and $y, L$ possesses the property $P_{n-1}\left(\mathcal{E}_{q}\right)$ at $x$ (Lemma 15). Moreover, $q$ must be equal to 2 (Lemma 15). 
By Lemma 16, there is a connected neighborhood $W$ of $x$ contained in $V$ and such that $W=T(W)$ and $W-L$ contains exactly two components $C$ and $T(C)$.

Since $W$ is contained in $Q, W-L^{\prime}$ has at least two components. Therefore $W \cap L^{\prime}=W \cap L$ and then $W-L^{\prime}$ has exactly two components $C$ and $T(C)$. Let $Q^{\prime}$ be a neighborhood of $x$ invariant under $H$ and contained in $W$. Then every element of $H$ maps $Q^{\prime} \cap C$ into $Q^{\prime} \cap T(C)$ or itself.

Suppose that the order of $H$ is $>2$. Then there is an element other than the identity maps $Q^{\prime} \cap C$ into itself. Certain power of this element, denoted by $T^{\prime}$, is of prime order $q$. Just as we have seen above, $q=2$ and there is a neighborhood $W^{\prime}$ of $x$ contained in $Q^{\prime}$ and such that $T^{\prime}\left(W^{\prime}\right)=W^{\prime}$ and $W^{\prime}-L^{\prime}$ has exactly two components $C^{\prime}$ and $T^{\prime}\left(C^{\prime}\right)$. $C^{\prime}$ is contained in either $C$ or $T(C)$; we may assume that $C^{\prime} \subset C$. Since $T^{\prime}\left(Q^{\prime} \cap C\right)=Q^{\prime} \cap C$, it follows that $T^{\prime}\left(C^{\prime}\right) \subset C$. Therefore $C \cup L^{\prime}$ contains $W^{\prime}$ and then contains $x$ as an inner point. This is clearly impossible.

8. Slices.

Definition. Let $X$ be a Hausdorff space and let $G$ be a compact Lie group acting topologically on $X$. Let $p$ be a point of $X$ and $G_{p}$ the isotropic subgroup of $G$ at $p$. A slice at $p$ is a subset $Y$ of $X$ containing $p$ and satisfying the following conditions:

(i) Whenever $g \in G$ and $x \in Y, g(x) \in Y$ if and only if $g \in G_{p}$.

(ii) If $Q$ is a small open cell which is a local cross-section to the cosets of $G_{p}$ at the identity of $G$, then $(g, x) \rightarrow g(x)$ defines a homeomorphism of $Q \times Y$ onto $Q(Y)$. Moreover, $Q(Y)$ is open in $X$.

Leмma 17. If $X$ is completely regular Hausdorff space and $G$ is a compact Lie group acting on $X$, then at every point of $X$ there exists a slice.

This Lemma was first proved by Montgomery-Yang [3] for complete metric spaces and then extended to completely regular spaces by Mostow [12].

\section{Theorem 2 .}

Theorem 2. Let $X$ be a ( $\mathfrak{B}, n)$-manifold and let $G$ be a compact Lie group acting topologically on $X$ such that the highest dimension of any orbit is $r$. Then for any integer $k, 0 \leqq k<r$, the union of all the orbits of dimension $\leqq k$ is a closed set of dimension $\leqq n-r+k-1$.

Proof. Let $X$ be a $(\mathfrak{B}, n)$-manifold and let $G$ be a compact Lie group acting on $X$. For each point $x$ of $X$, we denote by $G_{x}$ the isotropic subgroup of $G$ at $x$ and by $G_{x}^{*}$ the identity component of $G_{x}$. Then the order of the quotient group $G_{x} / G_{x}^{*}$ is finite and will be denoted by $m(x)$. 
For any integers $u \geqq 0, v \geqq 1$ we let

$$
X_{u, v}=\{x: x \in X, \operatorname{dim} G(x)=u, m(x)=v\}, \quad X_{u}=\bigcup_{v=1}^{\infty} X_{u, v} .
$$

Since every point $x$ of $X$ has a neighborhood $U$ such that whenever $y U, G_{y}$ is conjugate to a subgroup of $G_{x}[11]$, it follows that whenever $u \geqq 0$ and $v \geqq 0$,

$$
Z_{u, v}=X_{r} \cup X_{r-1} \cup \ldots \cup X_{u+1} \cup X_{u, 1} \cup X_{u, 2} \cup \ldots \cup X_{u, v}
$$

is open. Hence all $Z_{u, v}$ and consequently all $X_{u, v}$ are locally compact Hausdorff.

Each $X_{u, v}$ intersects $\bar{X}_{r}-X_{r}$ at a set of dimension $\leqq n-2$. In fact, let $p$ be a point of $X_{u, v} \cap\left(\bar{X}_{r}-X_{r}\right)$ and let $Y$ be a slice at $p$. Clearly $Y$ may be assumed to be connected and then is a ( $\mathfrak{B}, n-u)$-manifold (Lemma 3$)$. Since $p \in \bar{X}_{r}$, there is a point $x$ of $Y$ belonging to $X_{r}$. It follows from $\operatorname{dim} G_{p}>\operatorname{dim} G_{x}$ that there is a finite subgroup $H$ of $G_{p}$ in which the index of $H \cap G_{x}$ is $>2$. Making use of Theorem 1, one can easily show that the fixed point set of $H$ is of dimension $\leqq n-u-2$. Hence $X_{u, v} \cap Y$ is of dimension $\leqq n-u-2$, as it is contained in the fixed point set of $H$. Let $Q$ be an open $u$-cell in $G$ which contains the identity and is such that $(g, x) \rightarrow g(x)$ defines a homeomorphism of $Q \times Y$ onto $Q(Y)$ and that $Q(Y)$ is a neighborhood of $p$. Then $Q\left(X_{u, v} \cap Y\right)$ is a neighborhood of $p$ in $X_{u, v}$ and is of dimension $\leqq n-2$ (Lemma 5). Since $p$ is an arbitrary point of $X_{u, v} \cap\left(\bar{X}_{r}-X_{r}\right)$, the dimension of $X_{u, v} \cap\left(\bar{X}_{r}-X_{r}\right)$ is $\leqq n-2$ (Corollary to Lemma 4 ). From this result and Lemma 7 , it follows that the dimension of $\bar{X}_{r}-X_{r}$ is $\leqq n-2$. Hence $\bar{X}_{r}=X$ and consequently $X-X_{r}$, i.e., the union of all the orbits not of the highest dimension, is a closed set of dimension $\leqq n-2$, since otherwise $\bar{X}_{r}-X_{r}$ would be of dimension $n-1$ by Lemma 9.

Let $X^{*}$ be the orbit space of $X$ and let $f$ be the natural map of $X$ onto $X^{*}$. Then all $f\left(Z_{u, v}\right)$ are open in $X^{*}$. Hence all $f\left(Z_{u, v}\right)$ and consequently all $f\left(X_{u, v}\right)$ are locally compact Hausdorff. Let

$$
\operatorname{dim} f\left(X_{u, v}\right)=k_{u, v}, \quad 0 \leqq u<r .
$$

Let $p, Y, Q$ be as in the preceding paragraph. Then $f$ maps $X_{u, v} \cap Y$ onto a neighborhood of $f(p)$ in $f\left(X_{u, v}\right)$. Therefore $X_{u, v} \cap Y$ is of dimension $\leqq k_{u, v}$. Hence $Q\left(X_{u, v} \cap Y\right)$ is a neighborhood of $p$ in $X_{u, v}$ whose dimension is $\leqq k_{u, v}$ $+u$. Since $p$ is an arbitrary point of $X_{u, v}$, it follows that the dimension of $X_{u, v}$ is $\leqq k_{u, v}+u$. Hence our conclusion that $X_{0} \cup \cdots \cup X_{k}, 0 \leqq k<r$, is a closed set of dimension $\leqq n-r+k-1$ follows from Lemma 7 if we can show that

$$
k_{u, v} \leqq n-r-1, \quad 0 \leqq u<r .
$$


Suppose that our assertion is false. Then there is some $X_{\alpha, \beta}$ such that (i) $0 \leqq \alpha<r$, (ii) $k=k_{\alpha, \beta}>n-r-1$ and (iii) if $u>\alpha$ or if $u=\alpha$ and $v<\beta$, then $k_{u, v} \leqq n-r-1$. By Remark 3, there exist compact subsets $M, N$ of $X_{\alpha, \beta}$ such that $M \supset N$ and for some nonzero element $e$ of $H_{k}(M, N ; \mathfrak{B}), e \mid(M, K \cup N) \neq 0$ whenever $K$ is a proper compact subset of $(M-N)^{-}$. Let $p$ be a point of $f^{-1}(M-N)$ and let $Y$ be a connected slice at $p$. Clearly $G_{p}$ acts as a transformation group on $Y$ with $f(Y)$ as the orbit space. Let $A$ be a neighborhood of $p$ in $Y$ such that $G_{p}(A)=A$ and $f(A) \cap N=\varnothing$. Clearly for any neighborhood $B$ of $p$ in $Y$ contained in $A, e_{B}=e \mid(M, M-f(B)) \neq 0$.

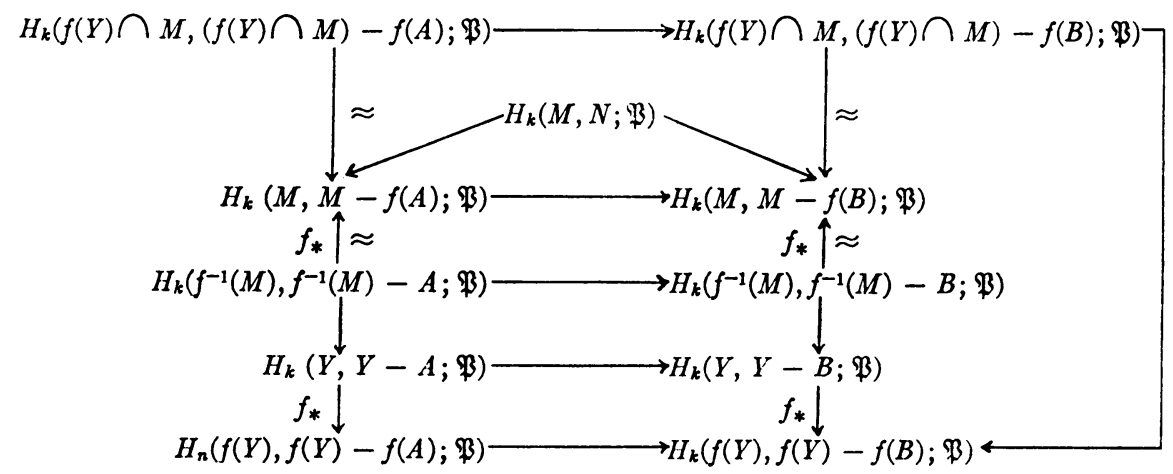

Let $e_{B}^{\prime}=f_{*}^{-1}\left(e_{B}\right) \mid(Y, Y-B)$; it is clear that $e_{B}^{\prime}=e_{A}^{\prime} \mid(Y, Y-B) . k$ is $\leqq n-u-2$, as $X_{u, v}$ is of dimension $\leqq n-2$. Since $Y$ is a $(\mathfrak{P}, n-u)$-manifold, $B$ can be chosen such that $e_{B}^{\prime}=0$. Moreover, we may assumc that $G_{p}(B)=B$. Let $e_{B}^{\prime \prime}$ be the element of $H_{k}(f(Y) \cap M,(f(Y) \cap M)-f(B)$; $;)$ such that $e_{B}^{\prime \prime} \mid(M, M-f(B))=e_{B}$. Then

$$
e_{B}^{\prime \prime} \neq 0
$$

and

$$
e_{B}^{\prime \prime} \mid(f(Y), f(Y)-f(B))=f_{*}\left(e_{B}^{\prime}\right)=0 .
$$

Since $f(Y)$ is contained in $f\left(Z_{\alpha, \beta}\right)$ which is of dimension $k$ by lemma 7 , it follows that the natural homomorphism of $I I_{k}(f(Y) \cap M I,(f(Y) \cap M I)-f(B)$; $;)$ into $I_{k}(f(Y), f(Y)-f(B)$; $\$$ is is one-one. Ilence we have arrived at a contradiction. This completes the proof of Theorem 2.

10. Appendix.

Definition. Let $X$ be a locally compact Hausdorff space and let $(5$ be a compact abelian group. $X$ is said to possess the property $Q(\mathfrak{C})$ at $x$ if for every neighborhood $A$ of $x$ there is a neighborhood $B$ of $x$ contained in $A$ and with the property that whenever $y$ is a point of $B$ and $C$ is a neighborhood of $y$ 
contained in $B$, there is a neighborhood $D$ of $y$ contained in $C$ and such that for every $k$,

$$
H_{k}(X-C, X-A ;(\mathfrak{)}) \mid(X-D, X-B)=0 .
$$

Proposition. Let $X$ be a locally compact Hausdorff space and let $x$ be a point of $X$. If $X$ possesses the property $P_{n}(\mathbb{E})$ at $x$, then $X$ possesses the property $Q(\mathfrak{S})$ at $x$.

Proof. By hypothesis, there is a fundamental $(\mathbb{E}, n)$-pair $(U, S)$ with $U$ containing $x$. Let $A$ be a fixed neighborhood of $x$. Then there is a neighborhood $B$ of $x$ contained in $A \cap U$ and such that

$$
H_{n}(X, X-A ;(S)|(X, X-B) \subset S|(X, X-B)
$$

and for every $k \neq n$,

$$
H_{k}(X, X-A ;(\mathcal{E}) \mid(X, X-B)=0 .
$$

Let $y$ be a point of $B$ and let $C$ be a neighborhood of $y$ contained in $B$. Then there is a neighborhood $D$ of $y$ contained in $C$ and such that

$$
H_{n}(X, X-C ;()|(X, X-D) \subset S|(X, X-D)
$$

and for every $k \neq n$,

$$
H_{k}(X, X-C ; \mathfrak{E}) \mid(X, X-D)=0 .
$$

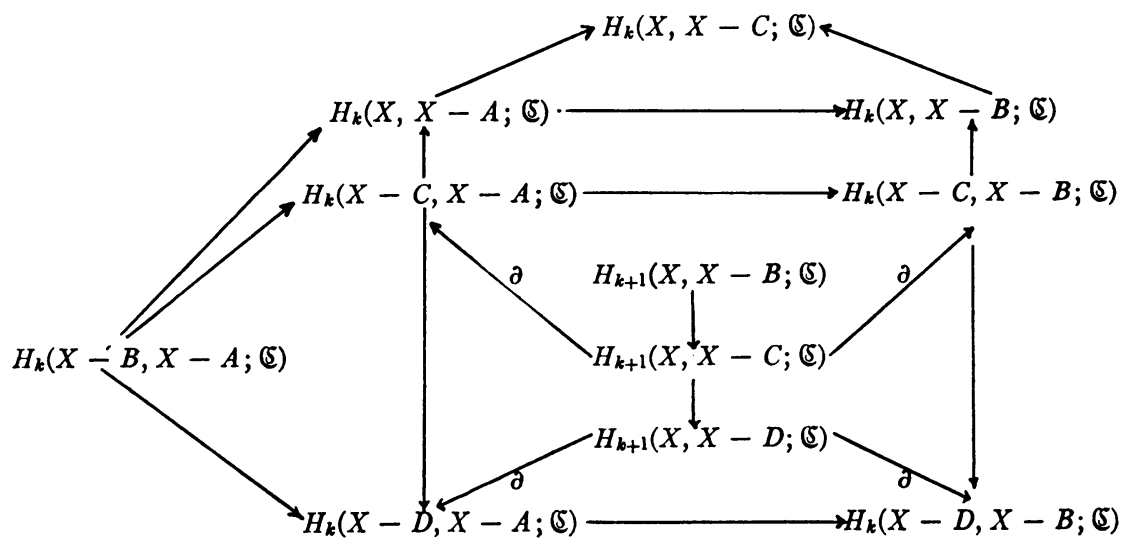

Let $e$ be an element of $H_{k}(X-C, X-A$; (5); then

$$
e \mid(X, X-B)=0 \text {. }
$$


In fact, if $k \neq n$, our assertion follows from (2). If $k=n$, it follows from (1) that for some $u \in S$,

$$
e|(X, X-B)=u|(X, X-B) .
$$

Since $e|(X, X-C)=0, u|(X, X-C)=0$ and then $u=0$. Hence $e \mid(X, X-B)$ $=0$.

From this result, it follows that for some $e^{\prime} \in H_{k}(X-B, X-A$; (5),

$$
e\left|(X, X-A)=e^{\prime}\right|(X, X-A) .
$$

Let

$$
\boldsymbol{\theta}^{\prime \prime}=e-e^{\prime} \mid(X-C, X-A) .
$$

Then

$$
e^{\prime \prime}|(X, X-A)=e|(X, X-A)-e^{\prime} \mid(X, X-A)=0 .
$$

Therefore for some $e^{\prime \prime \prime} \in H_{k+1}(X, X-C$; () ,

$$
\partial e^{\prime \prime \prime}=e^{\prime \prime} \text {. }
$$

Now we claim that

$$
\partial\left(e^{\prime \prime \prime} \mid(X, X-D)\right)=0,
$$

where $\partial$ is the boundary operator of the homology sequence of $(X, X-D$, $X-B)$. If $k \neq n-1$, then $e^{\prime \prime \prime} \mid(X, X-D)=0$ by (4) and therefore our assertion holds. If $k=n-1$, then by (3) there is some $u \in S$ such that

Hence our assertion again holds.

$$
\begin{aligned}
e^{\prime \prime \prime} \mid(X, X-D) & =u \mid(X, X-D) \\
& =(u \mid(X, X-B)) \mid(X, X-D) .
\end{aligned}
$$

From this result, we have

$$
\begin{aligned}
0 & =\partial\left(e^{\prime \prime \prime} \mid(X, X-D)\right) \\
& =e^{\prime \prime} \mid(X-D, X-B) \\
& =e \mid(X-D, X-B) .
\end{aligned}
$$

This proves our proposition.

\section{REFERENCES}

1. D. Montgomery, H. Samelson and L. Zippin, Singular points of a compact transformation group. Ann. of Math. (2) vol. 63 (1956) pp. 1-9.

2. D. Montgomery and C. T. Yang, Orbits of highest dimension, Trans. Amer. Math. Soc. vol. 87 (1958) pp. 284-294.

3. D. Montgomery and C. T. Yang, The existence of a slice, Ann. of Math. (2) vol. 65 (1957) pp. 108-116.

4. D. Montgomery, H. Samelson and C. 'T. Yang, Exceptional orbits of highest dimension, Ann. of Math. (2) vol. 64 (1956) pp. 131-141. 
5. P. A. Smith, Transformations of finite period, II. Ann. of Math. (2) vol. 40 (1939) pp. 690-711.

6. P. Alexandroff, On the dimension of normal spaces, Proc. Roy. Soc. London, Ser. A, vol. 189 (1947) pp. 11-39.

7. R. L. Wilder, Topology of manifolds, Amer. Math. Soc. Colloquium Publications, vol. 32, 1949.

8. S. Eilenberg and N. Steenrod, Foundations of algebraic topology, Princeton University Press, 1952.

9. H. Cohen, $A$ cohomological definition of dimension for locally compact Hausdorff spaces. Duke Math. J. vol. 21 (1954) pp. 209-224.

10. S. Eilenberg and S. MacLane, Group extensions and homology, Ann. of Math. (2) vol. 43 (1942) pp. 757-831.

11. D. Montgomery and L. Zippin, Topological transformation groups, Interscience Publishers, Inc., 1955.

12. G. D. Mostow, Equivariant imbeddings and euclidean spaces, Ann. of Math. vol. 65 (1957) pp. 432-446.

INSTITUTE FOR ADVANCED STUdY,

Princeton, New Jersey 